\title{
GABARAPL1 suppresses metastasis by counteracting PI3K/Akt pathway in prostate cancer
}

\author{
Wei Su ${ }^{1, *}$, Shibao $\mathrm{Li}^{2, *}$, Xiaofan Chen ${ }^{3}$, Lingyu Yin ${ }^{2}$, Ping Ma ${ }^{2}$, Yingyu Ma ${ }^{4}$, Bing Su${ }^{5,6}$ \\ ${ }^{1}$ Department of Orthopedics, The Third Affiliated Hospital, Xinxiang Medical University, Xinxiang, Henan, China \\ ${ }^{2}$ Department of Laboratory Medicine, The Affiliated Hospital of Xuzhou Medical College, Xuzhou, Jiangsu, China \\ ${ }^{3}$ Biomedical Research Institute, Shenzhen-PKU-HKUST Medical Center, Shenzhen, Guangdong, China \\ ${ }^{4}$ Department of Pharmacology and Therapeutics, Roswell Park Cancer Institute, Buffalo, NY, USA \\ ${ }^{5}$ Xinxiang Key Lab of Translational Cancer Research, The Third Affiliated Hospital, Xinxiang Medical University, Xinxiang, \\ Henan, China \\ ${ }^{6}$ Department of Epidemiology and Environmental Health, School of Public Health and Health Professions, University at \\ Buffalo, Buffalo, NY, USA \\ *These authors contributed equally to this work
}

Correspondence to: Yingyu Ma, email: yingyu.ma@roswellpark.org Bing Su, email: bsu2@buffalo.edu

Keywords: GABARAPL1, prostate cancer, metastasis, Akt, FOXOs

Received: August 15, $2016 \quad$ Accepted: December 02, 2016

Published: December 10, 2016

\section{ABSTRACT}

Metastasis remains the primary cause of prostate cancer (CaP)-related death. Using a genome wide shRNA screen, we identified GABARAPL1 as a potential CaP metastasis suppressor. GABARAPL1 mRNA levels inversely correlate with the invasive potential of a panel of human CaP cell lines. Lower mRNA levels correlate with higher Gleason scores in clinical CaP tumor samples. Moreover, Kaplan-Meier curves analysis showed that GABARAPL1 down-regulation in cancer tissues is associated with decreased disease-free survival in CaP patients. Knockdown of GABARAPL1 in human LNCaP cells results in increased invasion in vitro and lymph node metastasis in vivo. Vice versa, ectopic expression of GABARAPL1 decreases the invasiveness of CWR22Rv1 cells. Our previous in vitro shRNA screening identified FOXO4, a PI3K/ Akt-inactivating downstream target, as a potential CaP metastasis suppressor. We show here that silencing FOXOs leads to reduced GABARAPL1 expression and enhanced invasion in LNCaP cells. Transfection of constitutively-activated Akt (myrAkt) increased the invasion of LNCaP cells, which is associated with the inactivation of FOXOs and decreased GABARAPL1 expression. Indeed, forced expression of GABARAPL1 reversed the increased invasiveness of LNCaP/myr-Akt cells. Finally, immunohistochemistry analysis shows that Akt phosphorylation is negatively correlated with GABARAPL1 expression in human CaP tissues. Taken together, our data indicate that the suppression of FOXOs-GABARAPL1 signaling by Akt is an important mechanism for CaP progression and metastasis.

\section{INTRODUCTION}

Metastasis remains the primary cause of prostate cancer $(\mathrm{CaP})$-related death, which is the second leading cause of cancer deaths in men in the U.S. [1]. Unfortunately, no effective therapy is currently available $[2,3]$.

$\mathrm{PI} 3 \mathrm{~K} /$ Akt pathway is a major contributor to $\mathrm{CaP}$ progression $[4,5]$. In $42 \%$ of the primary $\mathrm{CaP}$ lesions and $100 \%$ of the metastatic tumors, PI3K/Akt pathway exhibits alterations (mutations/deletions, copy number variations, differential gene expression) in one or more components [6]. Because of the critical role of the PI3K/Akt pathway in promoting cancer progression, many inhibitors targeting this pathway are invented and tested in clinical trials [7]. Unfortunately, the therapeutic efficacy of these inhibitors remains modest [8]. Identifying genes that regulate the metastatic cascade will lead to novel and more effective treatment 
for the deadly metastatic $\mathrm{CaP}$ and prolong survival of CaP patients.

In order to understand the genetic components necessary for CaP metastasis, we used a high throughput shRNA screening approach to identify potential metastasis suppressors in the LNCaP orthotopic CaP model in vivo. Using DNA sequence analysis and the BLAST databank search, we identified gamma-aminobutyric acid (GABA) A receptor-associated protein-like 1 (GABARAPL1) as a metastasis suppressor candidate. GABARAPL1, also known as autophagy-related 8 (ATG8) or Glandular epithelial cell protein 1 (GEC1), belongs to the GABARAP family [9]. GABARAPL1 plays important roles in protein interaction and transportation, as well as autophagy, cell proliferation and tumor progression [9].

Previously, we used this functional shRNA screening approach in an invasion assay in vitro and identified FOXO4, a PI3K/Akt-inactivating downstream target, as a potential CaP metastasis suppressor $[10,11]$. Interestingly, several groups showed that GABARAPL1 expression is regulated by FOXOs [12-14], indicating that GABARAPL1 is a downstream target of FOXOs. Moreover, a recent study showed that Akt decreased the expression of GABARAPL1 [15]. Therefore, we hypothesized that the anti-metastatic GABARAPL1 may be involved in PI3K/Akt pathway. We next sought to determine the effect of activated Akt on the expression of FOXOs and GABARAPL1 and cell invasion in LNCaP cells. Our data strongly indicate that the suppression of FOXOs-GABARAPL1 signaling by Akt may be an important mechanism for $\mathrm{CaP}$ progression and metastasis.

\section{RESULTS}

\section{Genome-wide shRNA screening in LNCaP orthotopic CaP model identified GABARAPL1 as a potential metastasis suppressor}

To better understand the genetic components necessary for $\mathrm{CaP}$ metastasis, we used a high throughput genome-wide shRNA screening approach to identify genes suppressing lymph node metastasis in the LNCaP orthotopic $\mathrm{CaP}$ model. A schematic summary of the in vivo shRNA library screening of potential metastasis suppressor genes is presented in Figure 1A. We aimed to select for metastatic cells in regional lymph nodes from low invasive LNCaP cells, which were infected with GFPlabeled lentivirus encoding human genome-wide shRNA library ( 10,000 shRNAs). Stable clones were established and orthotopically injected to the prostates of male nude mice that have embedded testosterone pellets in the flanks. The growth of the primary tumors and pelvic local lymph node metastasis was assessed 10 weeks later by a bioluminescence imager. The primary tumors and lymph node metastasis were visible (Figure 1B-1C). Metastasis was not observed in lungs, livers and kidneys (Figure 1C).
Cells were isolated from the lymph nodes and puromycin resistant metastatic LNCaP cells were selected in vitro. Single colonies were established and PCR sequencing analysis with the bar codes and shRNA sequences followed by the BLAST databank searching (http://blast. ncbi.nlm.nih.gov/Blast.cgi) was performed to identify the genes that were silenced in these metastatic LNCaP cells by the shRNAs. These genes include GABA(A) receptor-assocated protein like 1 (GABARAPL1), Leucine proline-enriched proteoglycan (leprecan)1 (LEPRE1), Myosin IIIB (MYO3B), zinc finger protein 763 (ZNF763), tRNA aspartic acid methyltransferase 1 (TRDMT1), Opoid growth factor receptor-like 1 (OGFRL1), and Dyslexia susceptibility 1 candidate 1 (DYX1C1) (Table 1). Given the growing understanding of the association between autophagy and cancer progression and metastasis $[16,17]$, we selected GABARAPL1 to further investigate its potential as a metastasis suppressor.

\section{GABARAPL1 is downregulated in human metastatic CaP cell lines and $\mathrm{CaP}$ tumor tissues}

The expression levels of metastasis suppressor genes are often lower in highly metastatic tumor cells compared to poorly metastatic or non-metastatic tumor cells or normal cells [18]. To examine whether the expression pattern of GABARAPL1 is reversely related to metastasis potential, we first assessed GABARAPL1 mRNA expression in six $\mathrm{CaP}$ cell lines with various metastatic tendency and an immortalized human primary prostate epithelial cell RWPE-1 by qRT-PCR. GABARAPL1 expression is high in RWPE-1 and LNCaP cells (Figure 2A). More importantly, GABARAPL1 expression is inversely correlated with the metastatic ability of CaP cells (Figure 2A). LNCaP/LN3 and PC-3/ LN4, lymph node metastatic variants, showed markedly lower expression of GABARAPL1 compared to their parental lines LNCaP and PC-3, respectively (Figure 2A). Highly metastatic human CaP cell lines DU145 and CWR22Rv1 both have very low levels of GABARAPL1 (Figure 2A). The differences between RWPE-1 cells and $\mathrm{CaP}$ cell lines except for LNCaP cells are statistically significant $(P<0.001)$. Further, data mining in the Oncomine database (http://www.oncomine.org) including 4 individual studies revealed that GABARAPL1 is significantly down-regulated in metastatic CaP compared to primary tumor samples, and normal prostate tissue samples have the highest GABARAPL1 expression (Figure 2B).

To further investigate the observed inverse relationship of GABARAPL1 expression and metastasis in $\mathrm{CaP}$ cell lines, GABARAPL1 mRNA expression was examined in $80 \mathrm{CaP}$ tumor tissues and 59 benign prostate tissue samples from benign prostatic hyperplasia (BPH) patients by qRT-PCR. Overall, GABARAPL1 mRNA expression levels in $\mathrm{CaP}$ tumor tissues were significantly 
Table 1: Candidate metastasis suppressor genes identified from the genome-wide shRNA screening

\begin{tabular}{|c|c|c|c|}
\hline Gene symbol & Name & Accession & Biological functions \\
\hline LEPRE1 & $\begin{array}{l}\text { Leucine proline-enriched } \\
\text { proteoglycan(leprecan }) 1\end{array}$ & $\begin{array}{l}\text { NM_001042411, } \\
\text { NM_019782, } \\
\text { NM_019783, } \\
\text { NM_022356 }\end{array}$ & $\begin{array}{l}\text { cell growth, protein metabolic process, negative } \\
\text { regulation of cell proliferation, protein metabolic } \\
\text { process }\end{array}$ \\
\hline MYO3B & Myosin IIIB & $\begin{array}{l}\text { NM_001083615, } \\
\text { NM_138995 }\end{array}$ & $\begin{array}{l}\text { nucleotide binding, motor activity, actin binding, } \\
\text { protein serine/threonine kinase activity, ATP binding, } \\
\text { transferase activity }\end{array}$ \\
\hline ZNF763 & zinc finger protein 763 & $\begin{array}{l}\text { AK092240, } \\
\text { NM_001012753, } \\
\text { XM_001131183 }\end{array}$ & $\begin{array}{l}\text { nucleic acid binding, DNA binding, zinc ion binding, } \\
\text { metal ion binding }\end{array}$ \\
\hline TRDMT1 & $\begin{array}{l}\text { tRNA aspartic acid } \\
\text { methyltransferase } 1\end{array}$ & $\begin{array}{l}\text { NM_004412, } \\
\text { NM_176081, } \\
\text { NM_176083 }\end{array}$ & $\begin{array}{l}\text { DNA binding, RNA binding, DNA (cytosine-5-)- } \\
\text { methyltransferase activity, methyltransferase activity, } \\
\text { tRNA (cytosine-5-)-methyltransferase activity, } \\
\text { transferase activity }\end{array}$ \\
\hline GABARAPL1 & $\begin{array}{l}\mathrm{GABA}(\mathrm{A}) \quad \text { receptor- } \\
\text { assocated protein like } 1\end{array}$ & NM_031412 & $\begin{array}{l}\text { protein binding, beta-tubulin binding, GABA receptor } \\
\text { binding }\end{array}$ \\
\hline OGFRL1 & $\begin{array}{l}\text { Opoid growth factor } \\
\text { receptor-like } 1\end{array}$ & NM_024576 & receptor activity \\
\hline DYX1C1 & $\begin{array}{l}\text { Dyslexia susceptibility } 1 \\
\text { candidate } 1\end{array}$ & $\begin{array}{l}\text { NM_001033559, } \\
\text { NM_130810 }\end{array}$ & binding \\
\hline
\end{tabular}

\section{A}

Human whole-genome
shRNA library
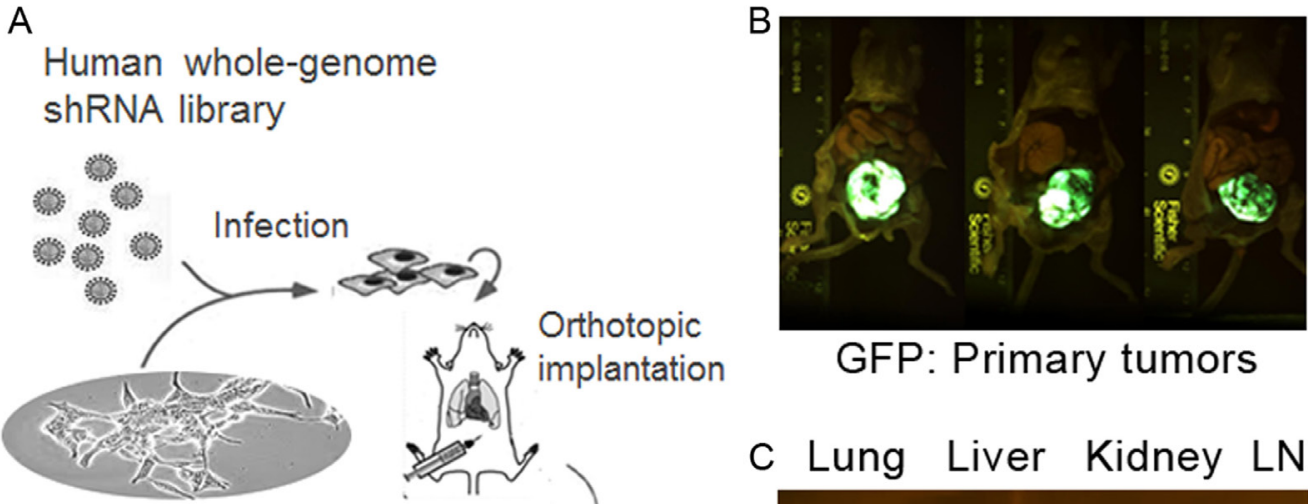

GFP: Primary tumors

C Lung Liver Kidney LN

$\mathrm{LNCaP}$ prostate

cancer cells

Lymph node metastasis

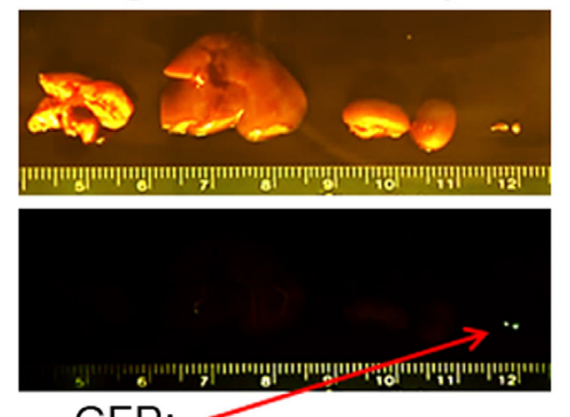

Light

\section{GFP:}

\section{Metastatic tumor cells}

PCR sequence

analysis and target

gene identification
Isolation of

metastatic cells
Fluorecence

Figure 1: Loss-of-function selection for metastasis-suppressor genes in the LNCaP CaP orthotopic xenograft model. (A) A schematic summary of the high-throughput genome-wide shRNA screening approach to identify genes suppressing regional lymph node metastasis in the LNCaP orthotopic metastatic model. (B) Sustained GFP fluorescence in the primary tumors formed after the injection of LNCaP cells infected with the DECODE (OpenBiosystems) pooled pGIPZ lentivirus library encoding human shRNAs. (C) A GFP fluorescence image of lymph node metastasis from one representative mouse. Red arrow indicates metastasis in the GFP image. 
lower than those in the benign prostate tissues $(P<0.001)$ (Figure $2 \mathrm{C}$ ). Among the $\mathrm{CaP}$ tumor tissues, a progressive loss of GABARAPL1 was found with increased Gleason scores (Figure 2C). Moreover, analysis of data from a published study including $174 \mathrm{CaP}$ cases [6], available on the cbioportal website (http://www.cbioportal.org/ public-portal/), revealed that 32 cases (18\%) that displayed GABARAPL1 downregulation in CaP tumor tissues had shorter disease free time compared with the 142 cases $(82 \%)$ whose GABARAPL1 expression levels were not altered in CaP tumor tissues (Figure 2D). These data strongly indicate that the loss of GABARAPL1 correlates with the aggressiveness of $\mathrm{CaP}$.

\section{Knock-down of GABARAPL1 promotes LNCaP cell invasion}

To determine whether the down-regulation of GABARAPL1 contributes to the highly metastatic capacity of $\mathrm{CaP}$ cells, LNCaP cells were transiently infected with two different shRNAs against GABARAPL1 and used to evaluate the impact on cell motility and invasiveness. Successful knockdown of GABARAPL1 protein expression was validated by Western blot analysis (Figure 3A). We next examined whether the knockdown of GABARAPL1 affects LNCaP cell motility using the scratch "wound" healing assay. LNCaP cells have poor motility and thus the wounded gap was only partially covered by shControl-transfected LNCaP cells in $24 \mathrm{~h}$ (Figure 3B). In contrast, either of the two shGABARAPL1-transfected LNCaP cells covered almost the entire damaged area by $24 \mathrm{~h}$ (Figure 3B), indicating that knockdown of GABARAPL1 promotes CaP cell motility. To further examine the involvement of GABARAPL1 in the invasion of CaP cells, LNCaP cells infected with shRNA-GABARAPL1 were used in the Matrigel-based invasion assay. shRNA-GABARAPL1 infection resulted in significantly $(P<0.05)$ increased invasion in LNCaP cells compared to control shRNA infection (Figure 3C). Conversely, overexpression of wild type-GABARAPL1 resulted in decreased invasiveness in CWR22Rv1 cells (Figure 3D). Taken together, these observations indicate that GABARAPL1 may be a potential metastasis suppressor.

\section{Loss of FOXOs down-regulates GABARAPL1 and promotes invasion in $\mathrm{LNCaP}$ cells}

To determine whether there is a relationship between FOXOs, GABARAPL1 and CaP invasion, LNCaP cells were transiently transfected with siRNA against three FOXOs (FOXO1, FOXO3 and FOXO4) or scrambled control siRNA, and subjected to Matrigel-based invasion assays. Three FOXO proteins were successfully knocked down as validated by Western blot analysis (Figure 3E). The knockdown of FOXOs resulted in decreased expression of GABARAPL1 (Figure 3E) and significant increase in the invasiveness of LNCaP cells $(P<0.05)$ (Figure $3 \mathrm{~F}$ ). These data suggest that FOXOs may suppress the invasiveness of $\mathrm{LNCaP}$ cells through regulating GABARAPL1 expression.

\section{Activation of Akt inhibits FOXOs and their target gene GABARAPL1 and promotes CaP invasion}

$\mathrm{PI} 3 \mathrm{~K} / \mathrm{Akt}$ pathway is a major contributor to $\mathrm{CaP}$ progression $[4,5]$. When PI3K/Akt pathway is activated in $\mathrm{CaP}$ cells, Akt directly phosphorylates FOXOs, which leads to FOXO inactivation and retention in the cytosol [19]. Akt activation also results in the down-regulation of GABARAPL1 [15]. Therefore, we addressed whether the activation of Akt could promote $\mathrm{LNCaP}$ invasiveness through the inactivation of FOXOs and their target gene GABARAPL1. The expression of constitutively-activated Akt (myr-Akt) promoted the phosphorylation of FOXO1 and FOXO3 and in the meantime decreased the expression of GABARAPL1 (Figure 3G). Moreover, the expression of constitutively activated Akt increased invasion of LNCaP cells (Figure 3H). Forced expression of GABARAPL1 blunted the myr-Akt-enhanced invasiveness of LNCaP cells (Figure 3I). Taken together, these data indicate that the activation of Akt inhibits FOXOs and their target gene GABARAPL1 and promotes CaP invasion.

\section{Akt expression is negatively related with GABARAPL1 expression in human CaP tumor tissues}

To investigate whether the expression of Akt and GABARAPL1 is correlated in clinical samples, we assessed their expression in ten pairs of primary $\mathrm{CaP}$ tumor tissues and matched adjacent non-tumor tissues by immunohistochemistry staining. Intense staining of p-Akt ${ }^{\mathrm{Ser} 473}$ was observed in $\mathrm{CaP}$ tumor tissues, while no positive staining was observed in adjacent non-tumor tissues (Figure 4A). In contrast, very low GABARAPL1 staining was observed in $\mathrm{CaP}$ tumor tissues compared to high expression in adjacent non-tumor tissues (Figure 4B). The relative GABARAPL1 expression histo-scores in tumor tissues and adjacent non-tumor tissues were calculated by the multiplication of the intensity and area of the IHC staining (Figure 4C). The finding that the expression of Akt and GABARAPL1 is inversely associated in $\mathrm{CaP}$ tumor tissues suggests that the inactivation of GABARAPL1 by Akt, most likely through FOXOs, is involved in $\mathrm{CaP}$ progression.

\section{DISCUSSION}

There is conclusive evidence that PI3K/Akt pathway plays critical roles in $\mathrm{CaP}$ progression and metastasis 


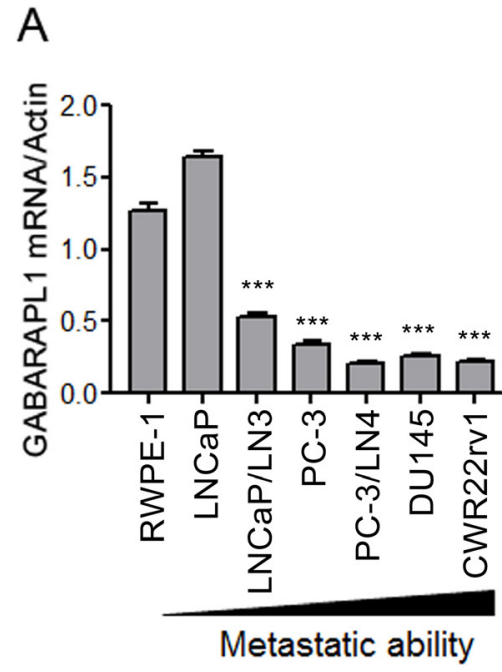

C

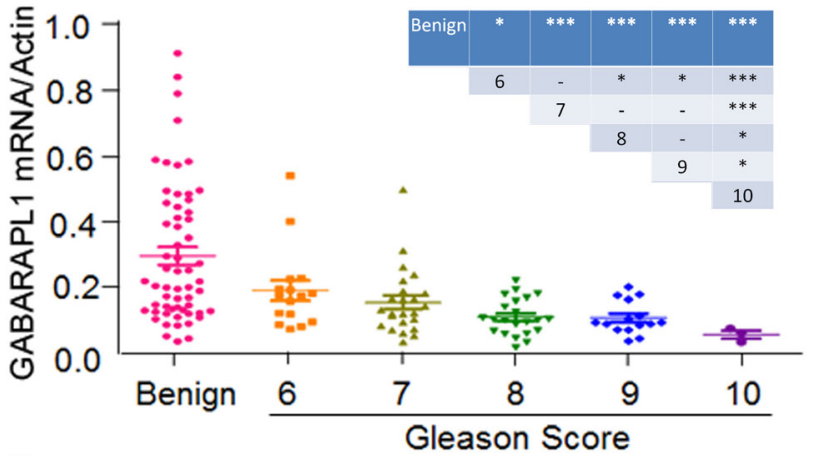

D

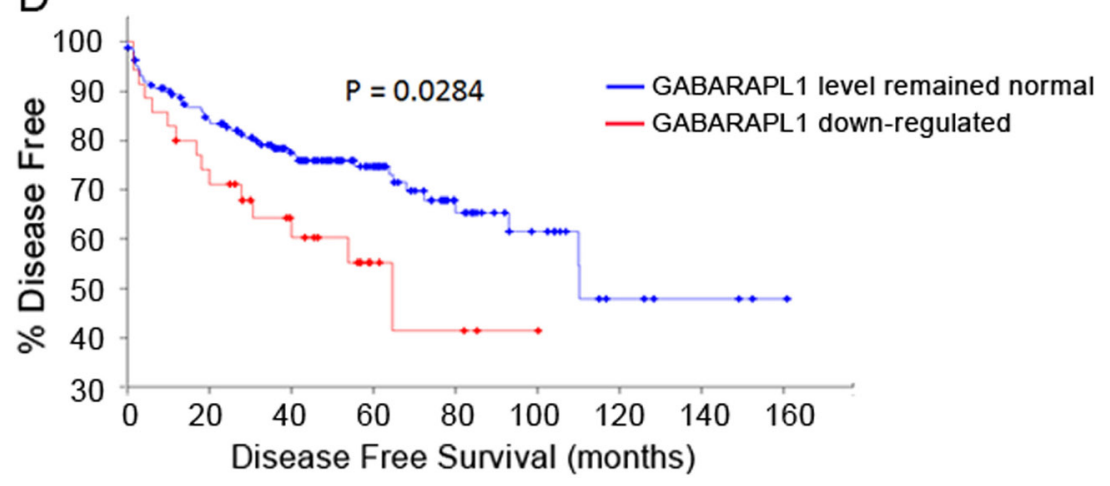

Figure 2: GABARAPL1 expression is inversely associated with invasiveness and metastatic in CaP cells and tissues. (A) GABARAPL1 expression in immortalized prostate epithelial cells RWPE-1 and CaP cell lines with different metastatic ability is assessed by qRT-PCR. Error bars, SE of three independent qRT-PCR assays. ${ }^{* * *} P<0.001$; cells indicated vs. RWPE-1 cells. (B) GABARAPL1 mRNA expression levels in normal (Nor), primary cancer (PCA) or metastases (MET). CaP tissues obtained from four studies available on the Oncomine website: Lapointe et al. [39], LaTulippe et al. [40], Taylor et al. [6], and Vanaja et al. [41]. (C) The expression of GABARAPL1 mRNA in a cohort of $80 \mathrm{CaP}$ tumor tissue samples, categorized by their Gleason scores, and 59 benign prostate tissue samples was assessed by qRT-PCR. $P$ values between specific two groups were showed in the inserted table: $* P<0.05 ; * * P<0.01$; ${ }^{* * *} P<0.001$. (D) Kaplan-Meier plot analysis (http://www.cbioportal.org/public-portal/) of disease free vs. time in $174 \mathrm{CaP}$ cases from a study conducted by Taylor et al. [6] is presented. 
[6]. As a result, many inhibitors targeting this pathway are now being tested in clinical trials. However, the therapeutic benefit of inhibitors of PI3K, Akt, or mTOR has been modest [8]. The treatment with anti-androgen receptor inhibitor, alone or in combination with $\mathrm{PI} 3 \mathrm{~K} /$ Akt/mTOR pathway inhibitor such as mTOR inhibitor, has been disappointing in castration-resistant prostate cancer (CRPC). In a recent phase II trial, the combination of carboplatin and everolimus, an mTOR inhibitor, showed minimal efficacy in metastatic CRPC [20]. These results suggest that other mechanisms may also be associated with $\mathrm{CaP}$ metastasis.

Vogelstein's group presented a surprising finding that genetic inactivation of both Akt1 and Akt 2 resulted in a striking decrease in liver metastasis in a colon cancer mouse model, which correlated with the activation of FOXO proteins, but not the well-known downstream signaling target GSK3b nor mTOR [21]. In line with this finding, our previous in vitro screening identified FOXO4 as a metastatic suppressor through the disruption of the PI3K/Akt pathway [10]. The FOXO family members, FOXO1, FOXO3 and FOXO4, are ubiquitously expressed transcription factors that function as tumor suppressors through inhibiting the expression of genes promoting proliferation, survival or de-differentiation [22, 23]. Increasing evidence supports anti-tumor roles for FOXO members in $\mathrm{CaP}$. For instance, the loss of FOXO3a promotes cancer formation in the TRAMP CaP mouse

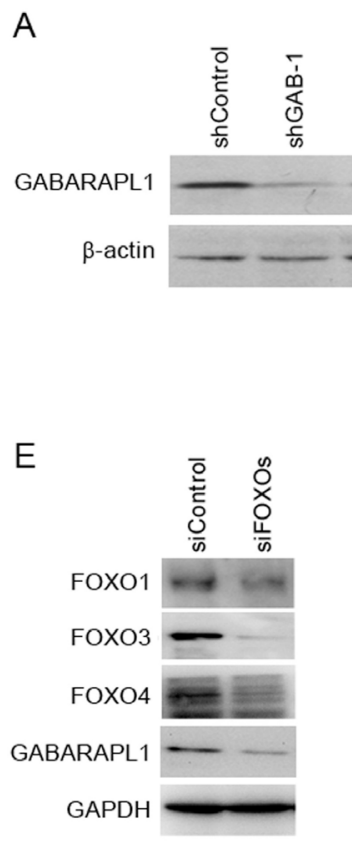

B

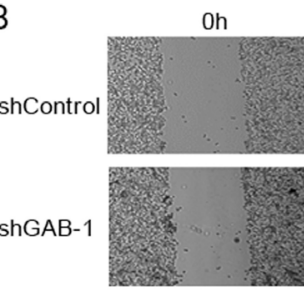

shGAB-2

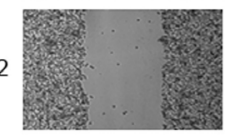

$\mathrm{F}$

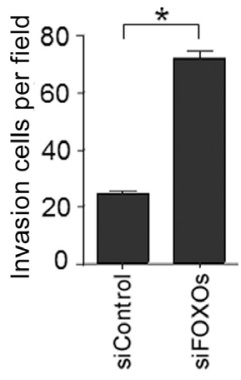

G

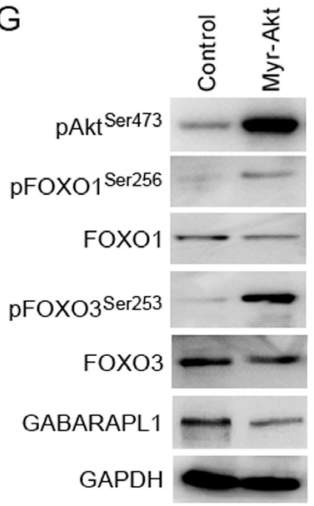

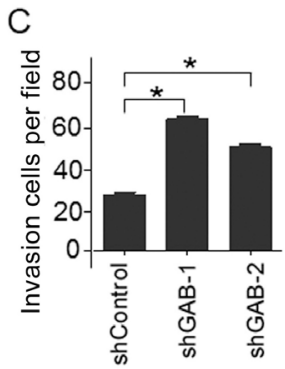

$\mathrm{D}$

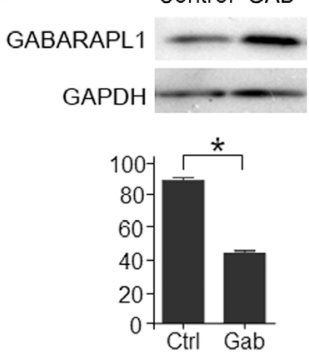

$\mathrm{H}$

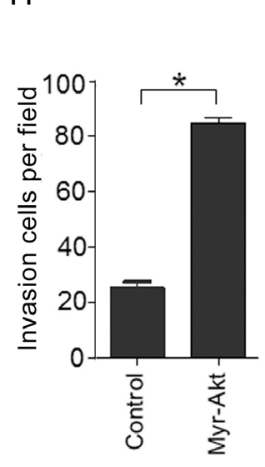

I

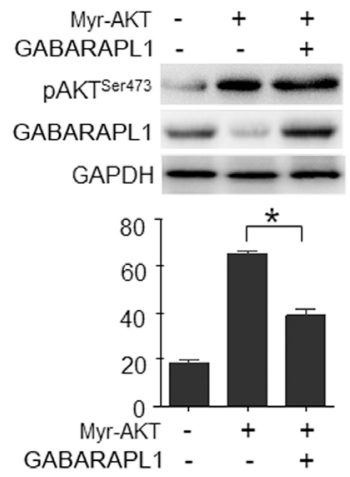

Figure 3: LNCaP cell invasion is promoted by Akt-mediated inactivation of FOXOs and the expression of FOXOsinduced gene GABARAPL1. (A) GABARAPL1 expression was knocked down by shRNAs and validated using IB analysis. $\beta$-actin was the internal control. (B) Wounds were introduced by scratching confluent monolayers of LNCaP cells transfected with shControl, shGAB-1 or shGAB-2. Migration was monitored by light microscopy at 0 and $24 \mathrm{~h}$. (C) Matrigel-based invasion assay was performed in LNCaP cells transfected with shControl, shGAB-1 or shGAB-2. The cell numbers per field were counted and the results summarized in a bar graph. Error bars, S.E. of triplicate experiments. ${ }^{*} P<0.05$. (D) Ectopic expression of wild type-GABARAPL1 decreases CWR22Rv1 invasiveness. Protein expression of GABARAPL1 was assessed by IB analysis (upper panel). The effect of overexpression of GABARAPL1 on Matrigel invasiveness was quantified (lower panel). Error bars, S.E. of triplicate experiments. $* P<0.05$. (E) FOXOs (FOXO1, FOXO3 and FOXO4) were knocked down by siRNA transfections in LNCaP cells. Protein expression of FOXOs and GABARAPL1 was assessed by IB analysis. GADPH was the internal loading control. (F) LNCaP cells with FOXOs knockdown were subjected to Matrigel-based invasion assays. The cell numbers per field were counted and the results summarized in a bar graph. Error bars, S.E. of triplicate experiments. ${ }^{*} P<0.05$. (G) LNCaP cells were transiently transfected with vectors harboring constitutively activated Akt (myr-Akt). Expression of phosphor-Akt (Ser473), phosphor-FOXO1 (Ser256), FOXO1, phosphor-FOXO3 (Ser253), FOXO3, and GABARAPL1 was assessed by IB analysis. GADPH was the loading control. (H) LNCaP cells transfected with myr-Akt or control vector were subjected to Matrigel-based invasion assays. The cell numbers per field were counted and the results summarized in a bar graph. Error bars, S.E. of triplicate experiments. ${ }^{*} P$ $<0.05$. (I) LNCaP cells were transiently transfected with control vector, myr-Akt or myr-Akt plus GABARAPL1. Expression levels of pAkt (Ser473) and GABARAPL1 were assessed by IB analysis. GAPDH was the loading control (upper panel). Matrigel invasion assay of LNCaP cells expressing control, myr-Akt or myr-Akt plus GABARAPL1 was presented in the lower panel. Error bars, S.E. of triplicate experiments. ${ }^{*} P<0.05$. 
model [24], whereas the upregulation or activation of FOXO proteins leads to growth arrest and apoptosis in CaP cells [25-27]. In contrast, Akt directly phosphorylates FOXO family members, thereby antagonizing the functions of FOXOs by promoting their association with 14-3-3 proteins and thus preventing their nuclear translocation [28]. The cytosol retention of FOXOs leads to their ubiquitylation-mediated proteasome degradation [29]. These observations indicate that Akt/FOXOs pathway may be associated with $\mathrm{CaP}$ progression and metastasis.

Our current study identified GABARAPL1 as a potentially metastasis suppressor using a genome wide shRNA screen for increased LNCaP lymph node metastasis in vivo. Growing findings indicate that GABARAPL1 is a negative regulator of cancer progression. Decreased GABARAPL1 expression has been reported in various cancer tissues compared to normal tissues [30]. GABARAPL1 expression level is associated with higher histological grade and lower risk of metastasis in breast adenocarcinoma patients, specifically for lymph node-positive patients [31]. These data suggest that GABARAPL1 may play a novel autophagy-independent role as a tumor metastasis suppressor, although it was first identified as an autophagy-related gene.

Our results further show that silencing FOXOs leads to decreased GABARAPL1 expression and increased invasion in LNCaP cells. Constitutivelyactivated Akt (myr-Akt) increased the invasion of LNCaP cells associated with inactivation of FOXOs and decreased GABARAPL1. Indeed, forced expression of GABARAPL1 partially reversed the increased invasiveness of $\mathrm{LNCaP} / \mathrm{myr}-\mathrm{Akt}$ cells.

Recently, a growing body of work [32-35] has revealed that autophagy promotes multiple steps in the metastatic cascade, such as regulation of the epithelialmesenchymal transition (EMT), tumor cell migration and invasion. Future studies are needed to elucidate exactly how GABARAPL1 inhibits prostate cancer cell invasion through autophagy pathway. It is unsurprising that the GABARAPL1 inhibited-invasion has been linked to autophagy.

In summary, our in vitro and in vivo RNAi screening results strongly suggest that $\mathrm{CaP}$ metastasis is promoted by Akt-mediated inhibition of FOXOs activation and the expression of its downstream targets such as GABARAPL1. Hence, we hypothesize that the inhibition of FOXOs-GABARAPL1 signaling by Akt is an important mechanism for $\mathrm{CaP}$ progression and metastasis (Figure 5).

\section{MATERIALS AND METHODS}

\section{Antibodies and reagents}

The following primary antibodies (Ab) were used in this study: rabbit polyclonal Abs specific for pan-Akt (\#ab8805), p-Akt (Ser473) (\#ab81283), antiGABARAPL1 (\#ab86503), and SP rabbit HRP kit were purchased from Abcam (Cambridge, MA). Anti-FOXO1 (\#2880), FOXO3 (\#12829), FOXO4 (\#9472), p-FOXO1 (Ser256) (\#9461), and p-FOXO3a (Ser253) (\#9466) were from Cell Signaling Technology (Beverly, MA). Mouse monoclonal anti-GAPDH and $\beta$-actin was from Santa Cruz Biotechnology (Santa Cruz, CA).

\section{Cell culture}

Human CaP LNCaP cells infected with the DECODE (OpenBiosystems) pooled pGIPZ lentivirus library encoding human shRNAs were established as
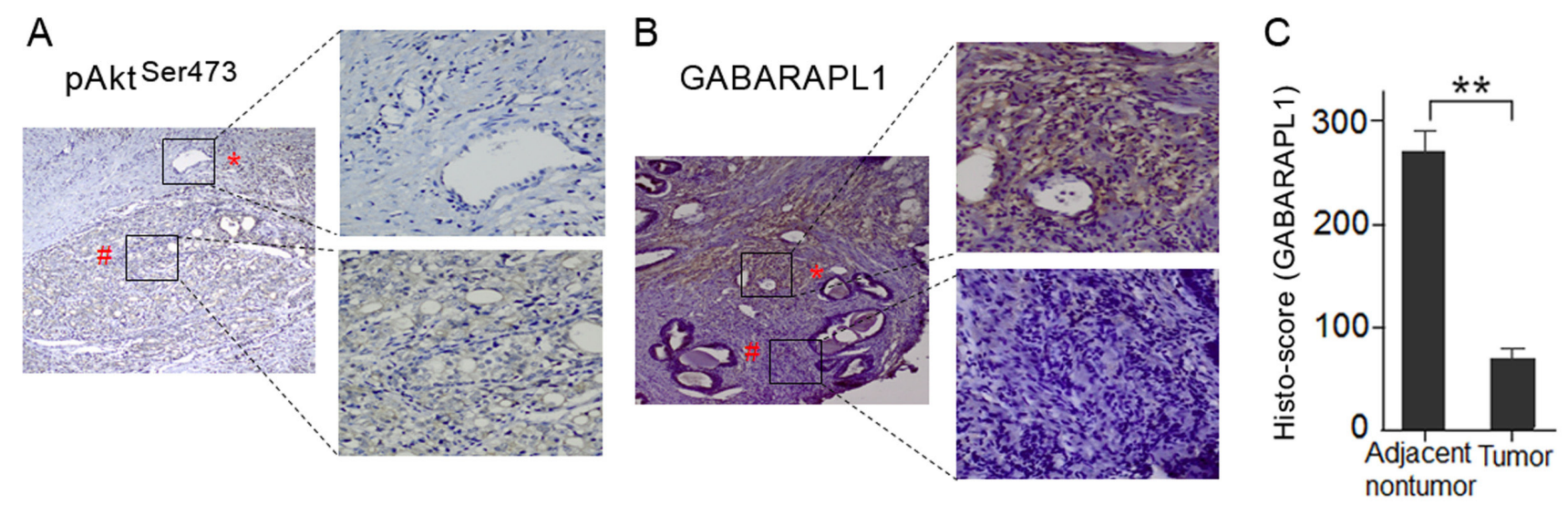

Figure 4: Akt expression is negatively correlated with GABARAPL1 expression in human CaP tumor tissues. (A) Phosphor-Akt (Ser473) level was assessed in primary human CaP tumor tissues and matched adjacent nontumor tissues by IHC. Representative images of the IHC study were presented. Magnification: image on the left, $\times 100$; images on the right, $\times 400$. (B) GABARAPL1 protein expression was examined in primary human $\mathrm{CaP}$ tumor tissues and matched adjacent nontumor tissues by IHC. Representative images of the IHC study were presented. Magnification: image on the left, $\times 100$; images on the right, $\times 400$. (C) The relative GABARAPL1 histoscores in tumor tissues and adjacent non-tumor tissues were calculated as described in Methods and presented in a bar graph. $* * P<0.01$. 
previously described [10]. LNCaP, PC-3, CWR22Rv1, RWPE-1, and DU145 were purchased from the Chinese Academy of Cell Bank. RWPE-1, LNCaP, LNCaP/LN3, and CWR22Rv1 cells were cultured in RPMI 1640 media supplemented with $10 \% \mathrm{FBS}$ and incubated at $37^{\circ} \mathrm{C}$ in a humidified incubator containing 5\% CO2. PC-3, and $\mathrm{PC}-3 \mathrm{M} / \mathrm{LN} 4$ cells were cultured in DMEM/F12 media supplemented with $10 \%$ FBS. DU145 cells were cultured in DMEM media supplemented with 10\% FBS.

\section{Clinical samples}

A total of $80 \mathrm{CaP}$ tissue samples and 59 control prostate tissue samples from BPH patients were obtained by needle biopsy in the Department of Urology in Huashan Hospital and the Department of Urology in Cancer Hospital affiliated with Fudan University (Shanghai, China) between March and September 2013. Ten pairs of primary tumor and matched adjacent non-tumor tissue samples were obtained from the Affiliated Hospital of Xuzhou Medical College (Xuzhou, Jiangsu, China). Written consents were obtained from all subjects prior to the recruitment. The study protocol was approved by the Ethics Committee of Huashan Hospital, Cancer Hospital of Fudan University, and the Affiliated Hospital of Xuzhou Medical College. All tumor samples were confirmed to contain more than $80 \%$ tumor cells by histological examination of sequential sections by pathologists. Staging was assessed after pathological examination of formalin fixed specimens according to the 1997 TNM classification. The clinical and pathologic characteristics of the subjects were reported previously [36].

\section{Transient transfection}

pLKO.1-shRNA-GABARAPL1 plasmid [37], constitutively-activated Akt1 (myr-Akt1) or control vectors were transiently co-transfected with pEGFP
DNA (Clontech/Takara, Mountainview, CA) into LNCaP cells using Lipofectamine reagent (Invitrogen, Carlsbad, CA) according to the manufacturer's protocol. pAdGABARAPL1 plasmid [37] was co-transfected transiently with pEGFP DNA into CWR22Rv1 cells. The transfected LNCaP and CWR22Rv1 cells were incubated for $40 \mathrm{~h}$. GFP positive cells were sorted by a fluorescence-activated cell sorter and used for Western blotting and invasion assays.

\section{Orthotopic CaP metastatic model}

Testosterone pellets were embedded in the flanks of 6-8 weeks old male nude mice (five mice per group) as previously described [10]. $10^{6}$ of LNCaP cells infected with GFP-labeled lentivirus library encoding human genomic shRNAs $(13,650$ targeted genes in 7 pools of 9,750 shRNA clones/pool) were orthotopically injected into the dorsal lobes of the prostate of these male nude mice as previously described [10]. Ten weeks later, mice were sacrificed and examined for the presence of GFP fluorescence in primary tumors and the organs using whole-body imaging (Lightools Research, Encinitas, CA). Pelvic lymph nodes were collected and digested with proteases. The cell suspension was placed on culture dishes and selected for puromycin $(2 \mu \mathrm{g} / \mathrm{ml})$ resistant metastatic LNCaP cells. All animal protocols were approved by the Institutional Animal Care and Use Committee of Shenzhen Biochemical Institute.

\section{siRNA transfection}

Synthetic ON-TARGETplus SMARTpool siRNA specific for FOXO1, FOXO3 or FOXO4, siCONTROL non-specific siRNA (NS-siRNA), and DharmaFECT-1 transfection reagent were purchased from Dharmacon (Lafayette, $\mathrm{CO}$ ). $\mathrm{LNCaP}$ cells were plated in 6-well plates $\left(5 \times 10^{4}\right.$ per well $)$ overnight. Cells were transfected with

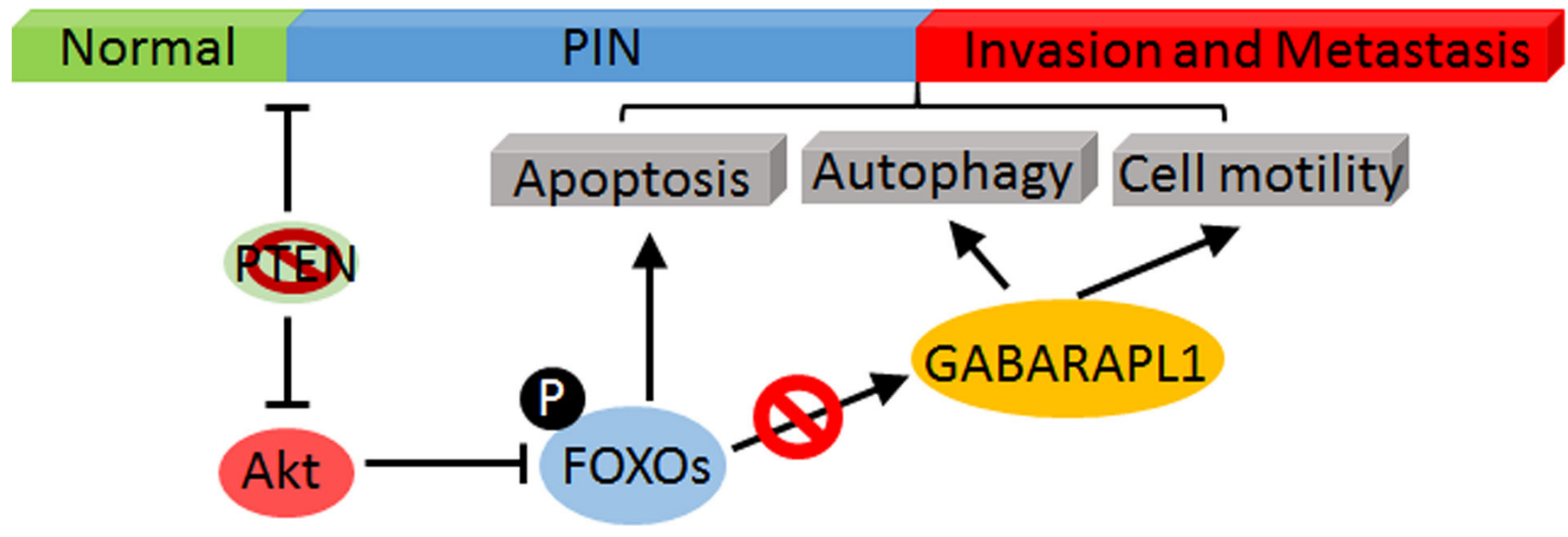

Figure 5: A schematic model of Akt-mediated negative regulation of FOXOs/GABARAPL1 in CaP progress and metastasis. Activated Akt directly phosphorylates FOXOs and leads to their inactivation, which subsequently inhibits the expression of their target gene GABARAPL1 and results in enhanced CaP invasion and metastasis. 
$50 \mathrm{nM}$ of NS-siRNA or three FOXO-specific siRNAs for $24 \mathrm{~h}$ using DharmaFECT1 following the manufacturer's protocol.

\section{Immunoblot (IB) analysis}

The cells were lysed in RIPA buffer with protease inhibitor cocktail (Roche Diagnostics, Mannheim, Germany). Protein samples $(40 \mu \mathrm{g})$ were separated by SDSPAGE, blotted onto polyvinylidene fluoride membranes which were blocked for $30 \mathrm{~min}$ with $5 \%$ bovine serum albumin (Sigma) in $1 \times \mathrm{TBS} / \mathrm{T}(0.1 \%$ Tween-20 in Trisbuffered saline) and then probed with primary antibodies as indicated. Digital imaging and signal quantification were performed on a Chemi-Genius2 Bio-Imager (Syngene, Frederick, MD) using GeneTools software.

\section{Invasion assay}

Modified Matrigel-based Boyden chamber assays were performed as previously described [38]. Briefly, LNCaP or CWR22Rv1 cells transfected with plasmids or siRNAs were plated in the inserts of the invasion chambers. FBS $(10 \%)$ was added as a chemoattractant. After $24 \mathrm{~h}$ of incubation, cells that had not penetrated the membranes were removed from the inserts with cotton swabs. Chambers were fixed and stained using Diff-Quik Stain Set (Dade Behring Inc., Newark, DE) and examined under a bright-field microscope. Numbers of invaded cells were counted in 6 fields per membrane $(\times 20$ objective $)$ and the average values of three independent experiments were presented.

\section{Immunohistochemistry (IHC) and imaging}

IHC assays were performed as previous described [36]. Briefly, ten pairs of primary tumor and matched adjacent non-tumor tissue samples were obtained from patients after prostatectomy and fixed in $10 \%$ buffered formalin, embedded in paraffin and sectioned at 4 microns. Slides were deparafinized in several baths of xylene and then rehydrated in graded alcohol series followed by ddH2O. Primary Abs to p-Akt(Ser473) (1:500) and GABARAPL1 (1:500) were diluted in 1\% BSA solution and incubated for $1 \mathrm{~h}$ at $37^{\circ} \mathrm{C}$, followed by staining using HRP-conjugated secondary antibody for $1 \mathrm{~h}$ at room temperature. Slides were scanned with Olympus BX53 microscope and viewed using Cellsens Entry (Olympus). The intensity of immunoreactivity (intensity score) was judged on an arbitrary scale of 0-4: 0 , negative; 1 , weak; 2 , moderate; 3 , strong and 4, very strong. Additionally, samples were given a percentage score based on the percentage of tissue area displaying each intensity score. A histo-score was calculated for each sample by multiplying the intensity score and the percentage score. Thus, the histo-score ranges from 0 to 400 .

\section{Quantitative reverse transcriptase PCR (qRT- PCR)}

Total RNA was isolated using TRIZOL Reagent (Sigma) and was reverse transcripted into cDNA using Revert Aid First Strand cDNA Synthesis Kit (Thermo Scientific). Real-time PCR was performed using SYBR premix EX Taq (TaKaRa) and analyzed with CFX96 Real-Time System (BIO-RAD). PCR primer sequences are as follows: GABARAPL1: forward, 5'-AGGAGGACCATCCCTTTGAGT-3'; and reverse, 5'-TGGCCAACAGTAAGGTCAGA-3'. $\beta$-actin: forward, 5'-GACCTGACTGACTACCTCATGAAGAT-3'; and reverse, 5'-GTCACACTTCATGATGGAGTTGAAG G-3'. $\beta$-actin was used as a housekeeping gene for the qRTPCR reactions. Each test was done in triple replication and the $2^{-\Delta \Delta C t}$ method [26] was used to calculate the expression of genes.

\section{Statistical analyses}

Statistical significances between groups were determined by two-tailed student's $t$-test. The differences in gene expression levels between $\mathrm{CaP}$ tumor tissues and $\mathrm{BPH}$ tissue specimens were tested by chi-square test. All statistical analyses were performed by using SPSS 16.0 software program. $P<0.05$ was considered statistically significant.

\section{Abbreviations}

CaP, prostate cancer; CRPC, castration-recurrent prostate cancer; GABARAPL1, gamma-aminobutyric acid (GABA) A receptor-associated protein-like 1; ATG8, autophagy-related 8; GEC1, Glandular epithelial cell protein 1; FOXOs, FOXO transcription factors; $\mathrm{BPH}$, benign prostate hyperplasia; LEPRE1, Leucine prolineenriched proteoglycan(leprecan)1; MYO3B, Myosin IIIB; ZNF763, zinc finger protein 763; TRDMT1, tRNA aspartic acid methyltransferase 1; OGFRL1, Opoid growth factor receptor-like 1; DYX1C1, Dyslexia susceptibility 1 candidate 1; qRT-PCR, real-time quantitative RT-PCR; LN, lymph nodes; PI3K, Phosphatidylinositol-3-kinase; IHC, Immunohistochemistry; myr-Akt1, Akt1 activation. EMT, epithelial-mesenchymal transition.

\section{ACKNOWLEDGMENTS}

We thank Dr. Irwin H. Gelman from Roswell Park Cancer Institute (Buffalo, New York, USA) for LNCaP prostate cancer cell lines infected with Decode RNAi viral libraries and myr-Akt1 plasmid. Dr. Ming Guan from Huashan Hospital (Shanghai, China) for prostate cancer tissues. The authors would like to thank Shenzhen Biomedical Research Support Platform for their excellent technical assistance. 


\section{CONFLICTS OF INTEREST}

The authors declare that they have no conflict of interest.

\section{GRANT SUPPORT}

The study was supported by the National Natural Science Foundation of China No. 81272380 (to B.S.), and No. 81572873 (to B.S.).

\section{REFERENCES}

1. Siegel R, Naishadham D, Jemal A. Cancer statistics, 2013. CA Cancer J Clin. 2013; 63:11-30. doi: 10.3322/ caac. 21166.

2. Karantanos T, Corn PG, Thompson TC. Prostate cancer progression after androgen deprivation therapy: mechanisms of castrate resistance and novel therapeutic approaches. Oncogene. 2013; 32:5501-11. doi: 10.1038/ onc.2013.206.

3. Feldman BJ, Feldman D. The development of androgenindependent prostate cancer. Nat Rev Cancer. 2001; 1:34-45. doi: 10.1038/35094009.

4. Bitting RL, Armstrong AJ. Targeting the PI3K/Akt/mTOR pathway in castration-resistant prostate cancer. Endocr Relat Cancer. 2013; 20:R83-99. doi: 10.1530/ERC-12-0394.

5. Sarker D, Reid AH, Yap TA, de Bono JS. Targeting the PI3K/AKT pathway for the treatment of prostate cancer. Clin Cancer Res. 2009; 15:4799-805. doi: 10.1158/10780432.CCR-08-0125.

6. Taylor BS, Schultz N, Hieronymus H, Gopalan A, Xiao Y, Carver BS, Arora VK, Kaushik P, Cerami E, Reva B, Antipin Y, Mitsiades N, Landers $\mathrm{T}$, et al. Integrative genomic profiling of human prostate cancer. Cancer Cell. 2010; 18:11-22. doi: 10.1016/j.ccr.2010.05.026.

7. Yap TA, Garrett MD, Walton MI, Raynaud F, de Bono JS, Workman P. Targeting the PI3K-AKT-mTOR pathway: progress, pitfalls, and promises. Curr Opin Pharmacol. 2008; 8:393-412. doi: 10.1016/j.coph.2008.08.004.

8. Fruman DA, Rommel C. PI3K and cancer: lessons, challenges and opportunities. Nat Rev Drug Discov. 2014; 13:140-56. doi: 10.1038/nrd4204.

9. Le Grand JN, Chakrama FZ, Seguin-Py S, Fraichard A, Delage-Mourroux R, Jouvenot M, Boyer-Guittaut M. GABARAPL1 (GEC1): original or copycat? Autophagy. 2011; 7:1098-107. doi: 10.4161/auto.7.10.15904.

10. Su B, Gao L, Baranowski C, Gillard B, Wang J, Ransom R, Ko HK, Gelman IH. A genome-wide RNAi screen identifies FOXO4 as a metastasis-suppressor through counteracting PI3K/AKT signal pathway in prostate cancer. PLoS One. 2014; 9:e101411. doi: 10.1371/journal.pone.0101411.

11. Kloet DE, Burgering BM. The PKB/FOXO switch in aging and cancer. Biochim Biophys Acta. 2011; 1813:1926-37. doi: 10.1016/j.bbamcr.2011.04.003.
12. Sengupta A, Molkentin JD, Yutzey KE. FoxO transcription factors promote autophagy in cardiomyocytes. J Biol Chem. 2009; 284:28319-31. doi: 10.1074/jbc.M109.024406.

13. Chiacchiera F, Matrone A, Ferrari E, Ingravallo G, Lo Sasso G, Murzilli S, Petruzzelli M, Salvatore L, Moschetta A, Simone C. p38alpha blockade inhibits colorectal cancer growth in vivo by inducing a switch from HIF1alpha- to FoxO-dependent transcription. Cell Death Differ. 2009; 16:1203-14. doi: 10.1038/cdd.2009.36.

14. Liu HY, Han J, Cao SY, Hong T, Zhuo D, Shi J, Liu Z, Cao W. Hepatic autophagy is suppressed in the presence of insulin resistance and hyperinsulinemia: inhibition of FoxO1-dependent expression of key autophagy genes by insulin. J Biol Chem. 2009; 284:31484-92. doi: 10.1074/ jbc.M109.033936

15. Paula-Gomes S, Goncalves DA, Baviera AM, Zanon NM, Navegantes LC, Kettelhut IC. Insulin suppresses atrophy- and autophagy-related genes in heart tissue and cardiomyocytes through AKT/FOXO signaling. Horm Metab Res. 2013; 45:849-55. doi: 10.1055/s-0033-1347209.

16. Feitelson MA, Arzumanyan A, Kulathinal RJ, Blain SW, Holcombe RF, Mahajna J, Marino M, MartinezChantar ML, Nawroth R, Sanchez-Garcia I, Sharma D, Saxena NK, Singh N, et al. Sustained proliferation in cancer: Mechanisms and novel therapeutic targets. Semin Cancer Biol. 2015; 35:S25-54. doi: 10.1016/j. semcancer.2015.02.006.

17. Mowers EE, Sharifi MN, Macleod KF. Autophagy in cancer metastasis. Oncogene. 2016. doi: 10.1038/onc.2016.333.

18. Steeg PS. Metastasis suppressors alter the signal transduction of cancer cells. Nat Rev Cancer. 2003; 3:55-63. doi: $10.1038 / \mathrm{nrc} 967$.

19. Zhang Y, Gan B, Liu D, Paik JH. FoxO family members in cancer. Cancer Biol Ther. 2011; 12:253-9. doi:

20. Vaishampayan U, Shevrin D, Stein M, Heilbrun L, Land S, Stark K, Li J, Dickow B, Heath E, Smith D, Fontana J. Phase II Trial of Carboplatin, Everolimus, and Prednisone in Metastatic Castration-resistant Prostate Cancer Pretreated With Docetaxel Chemotherapy: A Prostate Cancer Clinical Trial Consortium Study. Urology. 2015; 86:1206-11. doi: 10.1016/j.urology.2015.08.008.

21. Ericson K, Gan C, Cheong I, Rago C, Samuels Y, Velculescu VE, Kinzler KW, Huso DL, Vogelstein B, Papadopoulos N. Genetic inactivation of AKT1, AKT2, and PDPK1 in human colorectal cancer cells clarifies their roles in tumor growth regulation. Proc Natl Acad Sci USA. 2010; 107:2598-603. doi: 10.1073/pnas.0914018107.

22. van der Vos KE, Coffer PJ. The extending network of FOXO transcriptional target genes. Antioxid Redox Signal. 2011; 14:579-92. doi: 10.1089/ars.2010.3419.

23. Lam EW, Brosens JJ, Gomes AR, Koo CY. Forkhead box proteins: tuning forks for transcriptional harmony. Nat Rev Cancer. 2013; 13:482-95. doi: 10.1038/nrc3539.

24. Shukla S, Bhaskaran N, Maclennan GT, Gupta S. Deregulation of FoxO3a accelerates prostate cancer 
progression in TRAMP mice. Prostate. 2013; 73:1507-17. doi: $10.1002 /$ pros.22698.

25. Chen Q, Ganapathy S, Singh KP, Shankar S, Srivastava RK. Resveratrol induces growth arrest and apoptosis through activation of FOXO transcription factors in prostate cancer cells. PLoS One. 2010; 5:e15288. doi: 10.1371/journal. pone. 0015288 .

26. Kikuno N, Shiina H, Urakami S, Kawamoto K, Hirata H, Tanaka Y, Place RF, Pookot D, Majid S, Igawa M, Dahiya R. Knockdown of astrocyte-elevated gene-1 inhibits prostate cancer progression through upregulation of FOXO3a activity. Oncogene. 2007; 26:7647-55. doi: 10.1038/sj.onc. 1210572.

27. van Duijn PW, Ziel-van der Made AC, van der Korput JA, Trapman J. PTEN-mediated G1 cell-cycle arrest in LNCaP prostate cancer cells is associated with altered expression of cell-cycle regulators. Prostate. 2010; 70:135-46. doi: 10.1002/pros.21045.

28. Yang JY, Hung MC. Deciphering the role of forkhead transcription factors in cancer therapy. Curr Drug Targets. 2011; 12:1284-90.

29. Calnan DR, Brunet A. The FoxO code. Oncogene. 2008; 27:2276-88. doi: 10.1038/onc.2008.21.

30. Nemos C, Mansuy V, Vernier-Magnin S, Fraichard A, Jouvenot M, Delage-Mourroux R. Expression of gec1/ GABARAPL1 versus GABARAP mRNAs in human: predominance of gec1/GABARAPL1 in the central nervous system. Brain Res Mol Brain Res. 2003; 119:216-9.

31. Berthier A, Seguin S, Sasco AJ, Bobin JY, De Laroche G, Datchary J, Saez S, Rodriguez-Lafrasse C, Tolle F, Fraichard A, Boyer-Guittaut M, Jouvenot M, DelageMourroux R, et al. High expression of gabarapl1 is associated with a better outcome for patients with lymph node-positive breast cancer. Br J Cancer. 2010; 102:1024 31. doi: 10.1038/sj.bjc.6605568.

32. Qiang L, Zhao B, Ming M, Wang N, He TC, Hwang S, Thorburn A, He YY. Regulation of cell proliferation and migration by $\mathrm{p} 62$ through stabilization of Twist1. Proc Natl Acad Sci USA. 2014; 111:9241-6. doi: 10.1073/ pnas.1322913111.

33. Lock R, Kenific CM, Leidal AM, Salas E, Debnath J. Autophagy-dependent production of secreted factors facilitates oncogenic RAS-driven invasion. Cancer Discov. 2014; 4:466-79. doi: 10.1158/2159-8290.CD-13-0841.

34. Kenific CM, Stehbens SJ, Goldsmith J, Leidal AM, Faure N, Ye J, Wittmann T, Debnath J. NBR1 enables autophagy-dependent focal adhesion turnover. J Cell Biol. 2016; 212:577-90. doi: 10.1083/jcb.201503075.

35. Sharifi MN, Mowers EE, Drake LE, Collier C, Chen H, Zamora M, Mui S, Macleod KF. Autophagy Promotes Focal Adhesion Disassembly and Cell Motility of Metastatic Tumor Cells through the Direct Interaction of Paxillin with LC3. Cell Rep. 2016; 15:1660-72. doi: 10.1016/j. celrep.2016.04.065.

36. Li S, Ma Y, Xie C, Wu Z, Kang Z, Fang Z, Su B, Guan M. EphA6 promotes angiogenesis and prostate cancer metastasis and is associated with human prostate cancer progression. Oncotarget. 2015; 6:22587-97. doi: 10.18632/ oncotarget.4088.

37. Xie CW, Zhou Y, Liu SL, Fang ZY, Su B, Zhang W. Gabarapl1 mediates androgen-regulated autophagy in prostate cancer. Tumour Biol. 2015; 36:8727-33. doi: 10.1007/s13277-015-3542-8.

38. Su B, Bu Y, Engelberg D, Gelman IH. SSeCKS/Gravin/ AKAP12 inhibits cancer cell invasiveness and chemotaxis by suppressing a protein kinase C- Raf/MEK/ERK pathway. J Biol Chem. 2010; 285:4578-86. doi: 10.1074/jbc. M109.073494.

39. Lapointe J, Li C, Higgins JP, van de Rijn M, Bair E, Montgomery K, Ferrari M, Egevad L, Rayford W, Bergerheim U, Ekman P, DeMarzo AM, Tibshirani R, et al. Gene expression profiling identifies clinically relevant subtypes of prostate cancer. Proc Natl Acad Sci USA. 2004; 101:811-6. doi: 10.1073/pnas.0304146101.

40. LaTulippe E, Satagopan J, Smith A, Scher H, Scardino P, Reuter V, Gerald WL. Comprehensive gene expression analysis of prostate cancer reveals distinct transcriptional programs associated with metastatic disease. Cancer Res. 2002; 62:4499-506. doi:

41. Vanaja DK, Cheville JC, Iturria SJ, Young CY. Transcriptional silencing of zinc finger protein 185 identified by expression profiling is associated with prostate cancer progression. Cancer Res. 2003; 63:3877-82. doi: 